\title{
Pyogenic Arthritis of the Facet Joint with Concurrent Epidural and Paraspinal Abscess: A Case Report
}

\author{
Kee-Won Rhyu, Sang-Eun Park, Jong-Hun Ji, In Park, Young-Yul Kim \\ Department of Orthopedic Surgery, Daejeon St. Mary's Hospital, \\ The Catholic University of Korea College of Medicine, Daejeon, Korea
}

\begin{abstract}
Pyogenic arthritis of lumber spinal facet joints is an extremely rare condition. There are only 40 reported cases worldwide. Most cases were associated with history of paravertebral injection, which was not found in our patient. At the time of hospital admission, he had no abnormal magnetic resonance image findings. Two weeks later, he developed pyogenic facet joint arthritis associated with paravertebral and epidural abscess. This report is the first to describe delayed presentation of pyogenic arthritis associated with paravertebral abscess and epidural infection.
\end{abstract}

Key Words: Zygapophyseal joint, Pyogenic arthritis, Lumbar spine

\section{Introduction}

To date, only 40 cases of pyogenic arthritis developed in the spinal facet joints have been reported worldwide. Furthermore, Magee et al. [1] reported a case of pyogenic arthritis in the spinal facet joint with simultaneous paraspinal muscle and epidural abscess. In Korea, Lee et al. [2] reported a similar case that developed following epidural steroid injection. However, there is no reported case of idiopathic pyogenic arthritis in the literature.

We herein report our experience of managing idiopathic pyogenic arthritis in the lumbar facet joint that resulted in paraspinal muscle abscess invading into the epidural space, and include a review of the literature.

\section{Case Report}

A 45-year-old male presented to our hospital with a one- week history of severe back pain, which became exacerbated 2 days prior to admission. Except for anemia, the patient did not have other past medical history. He was apyrexial on admission. The initial straight leg-raising test was negative. There were no other associated symptoms. His initial plain radiograph was unremarkable (Fig. 1). His leukocyte count (white blood cell count) was 14,800 cells per liter, erythrocyte sedimentation rate (ESR) was $120 \mathrm{~mm} / \mathrm{hr}$, and C-reactive protein (CRP) was elevated to 14.3 . T2 weighted-images of magnetic resonance image (MRI) performed at another hospital showed slightly high signal density within the paraspinal muscles, for which intravenous antibiotics were administered (Fig. 2). Technetium bone scan performed on day 3 of admission was unremarkable. Two weeks after admission, the patient began to experience paraesthesia in both lower extremities. Straight leg raising test became positive, and pain in the vicinity of the spine became worsened. Plain radiograph of the spine showed slight osteolytic lesion in the facet joint that was further

Received Jul 15, 2010; 1st Revised Nov 19, 2010; Accepted Nov 19, 2010

Corresponding author: Young-Yul Kim, MD

Department of Orthopedic Surgery, Daejeon St. Mary's Hospital,

520-2 Daeheung-dong, Jung-gu, Daejeon 301-723, Korea

Tel: +82-042-220-9530, Fax: +82-042-221-0429, E-mail: kimtwins72@paran.com 


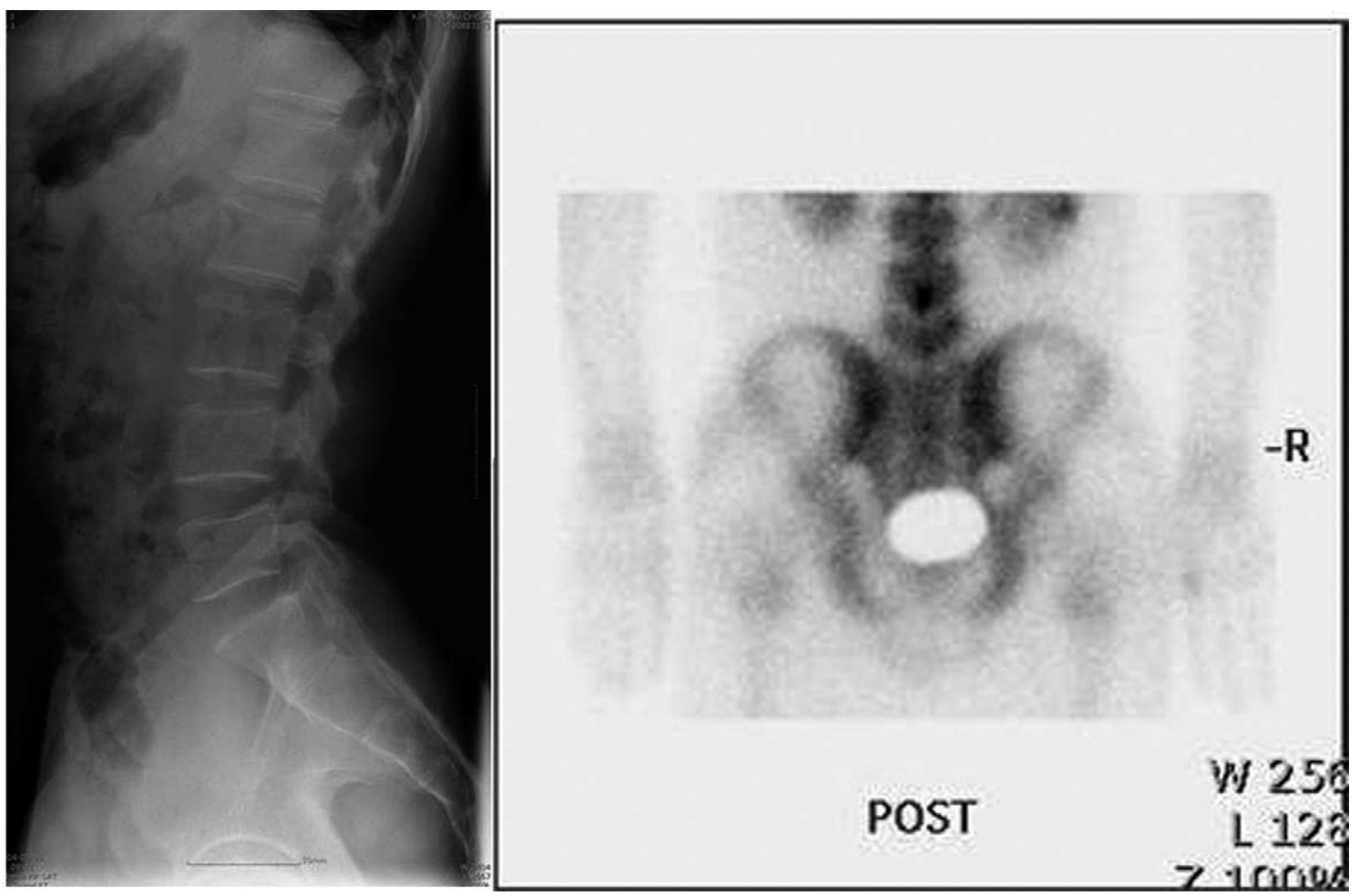

Fig. 1. Initial assessment of patient with plain radiograph, bone scan showed.

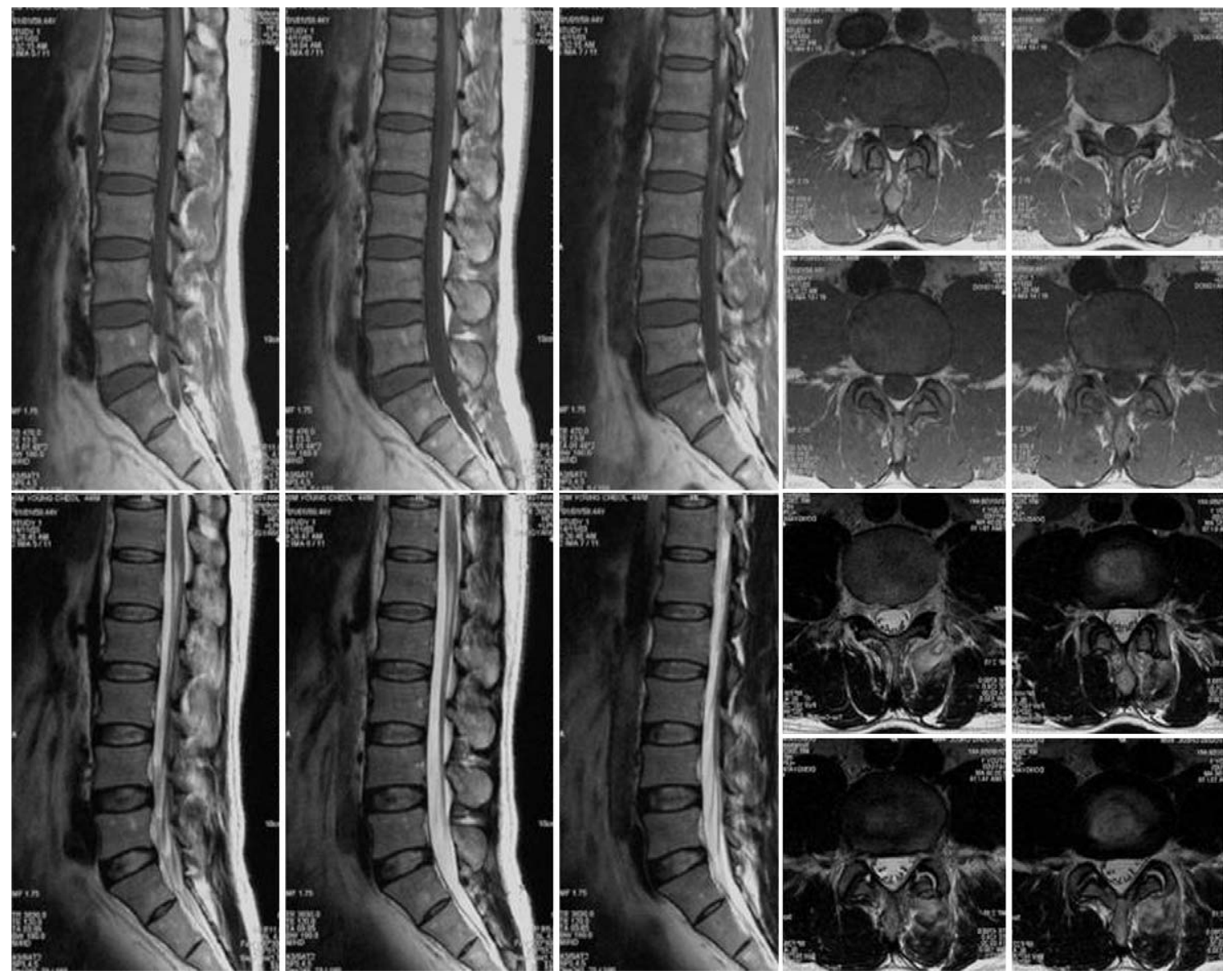

Fig. 2. Magnetic resonance image. Low-grade muscular enhancement was seen in axial images. 

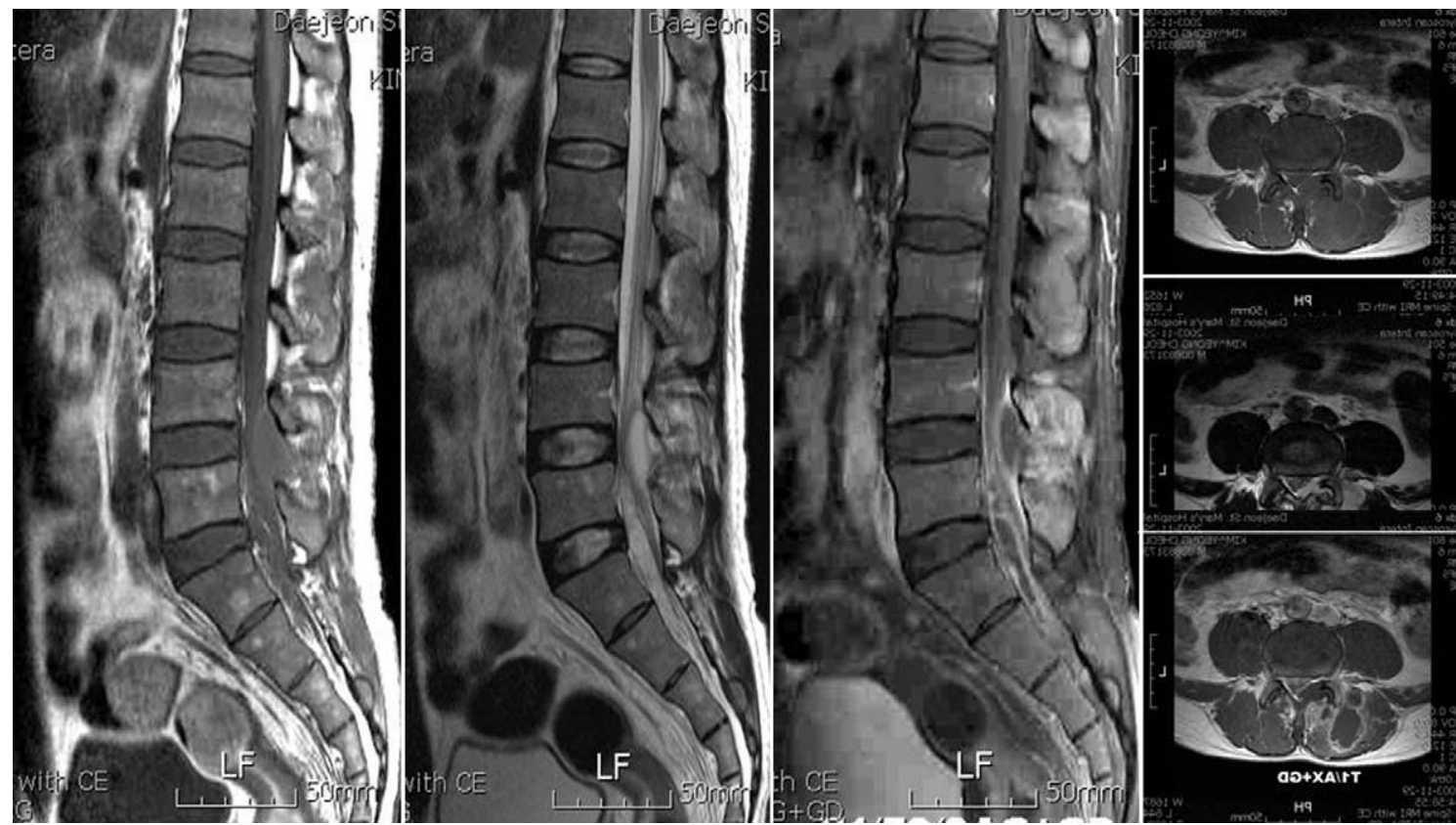

Fig. 3. Two weeks after admission, follow up magnetic resonance image was performed. Mild signal increase in L45 area and no evidence of pus collection in saggital and axial images. Gadolinium enhancement was identified.

investigated by MRI, which demonstrated a paraspinal muscle abscess in the L2-5 vertebrae invading into the right facet joint, causing osteo-destructive changes and associated with dilatation of the epidural space in the vicinity of the fourth lumbar vertebra. An abscess-like mass showed low signal intensity on $\mathrm{T} 1$ weighted images, high signal intensity on T2 weighted images, and marginal enhancement on Gadolium enhanced images. It compressed on the dura in the spinal canal (Fig. 3). Surgical debridement was performed in response to the patient's neurological symptoms. Intraoperatively, the paraspinal muscle abscess was drained between the L3-5 lumbar vertebrae. In addition, L4-5 laminectomy, right medial facetectomy, and posterior decompression were performed to remove the epidural abscess (Fig. 4). Post-operatively, the patient experienced improvement in his neurological symptoms. MRI performed 2 weeks post-surgery showed resolution of the pyogenic mass, although paraspinal high signal intensity persisted, for which the patient was managed with antibiotics for 3 further weeks. The bacterial strain cultured were Staphylococcus, albeit without information on resistance (Fig. 5). Repeat blood test performed 6 weeks after surgery was unremarkable, and treatment was subsequently terminated.

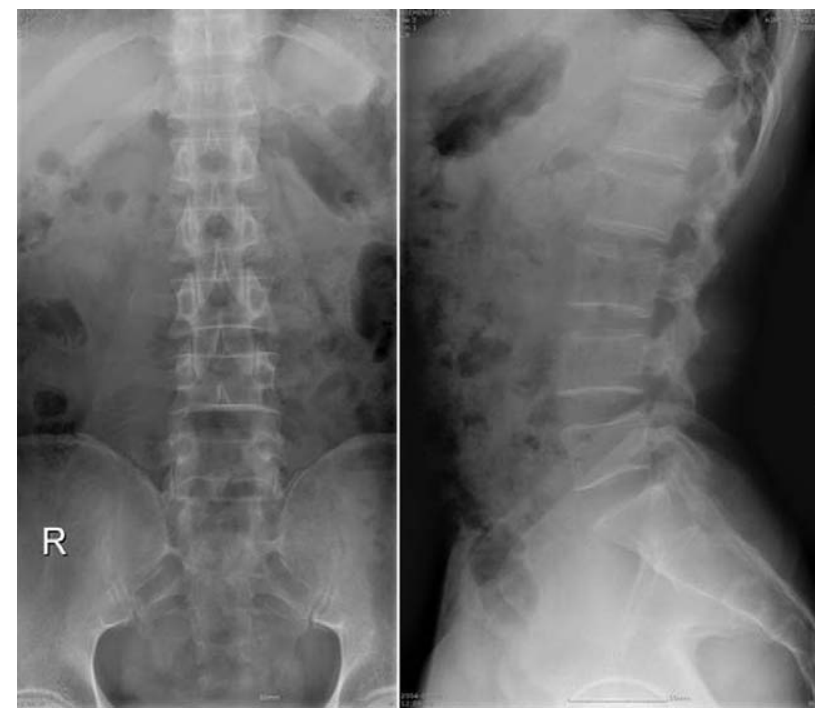

Fig. 4. We performed surgery debrided the pyogenic collection. The patient's symptom was improved post-operatively.

\section{Discussion}

Pyogenic arthritis in the spinal facet joint is a very rare condition. Until now, approximately 40 cases have been reported in the literature. Recently, the development of pyogenic arthritis in the spinal facet joint following steroid injection to the facet joint has been reported in the literature [2]. Nonetheless, we thought it would be valuable to discuss 


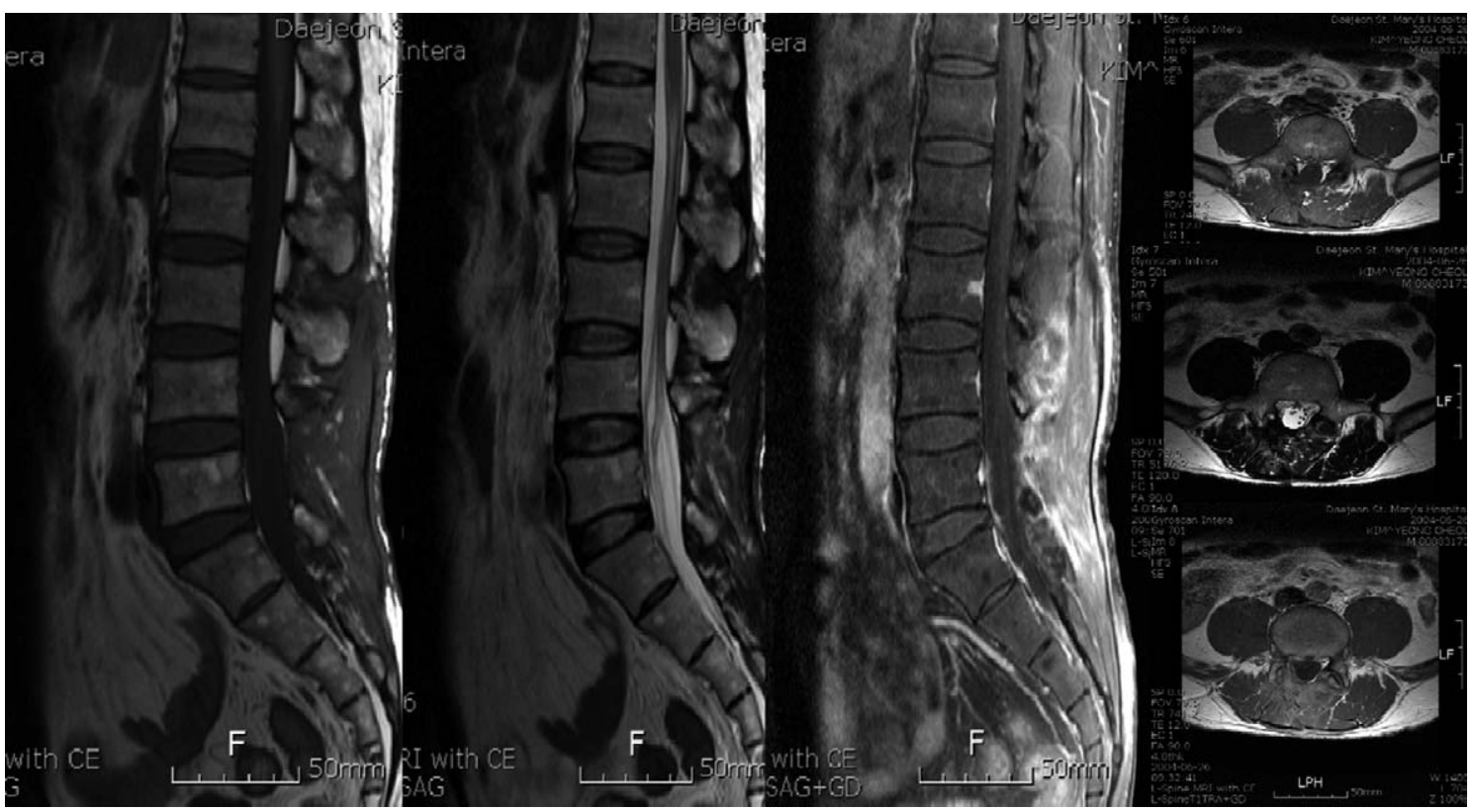

Fig. 5. Post-surgical magnetic resonance image (MRI). Postoperative follow up MRI showed no evidence of recurrent pyogenic abscesses.

our experience, because idiopathic pyogenic arthritis in the spinal facet joint is predominantly a secondary infection caused by pyogenic arthritis in the vertebral body. Nonetheless, recently, the concept is changing to hematogenous disseminating in more than half of the cases, and develop solely [3,4]. In addition, Muffoletto et al. [5] reported that in spinal infection, hematogenous pyogenic arthritis developed in 6 patients (4\%), the predominant causative bacteria were Staphylococcus aureus and S. epidermidis, and both ESR and CRP were elevated in all cases. Our patient was apyrexial at the time of hospital admission, although elevated ESR and CRP were in evident. As such, the cause was secondary to infection.

Characteristic symptoms of pyogenic facet joint arthritis include persistent lower back pain not relieved by rest and fever. It has been reported that $90 \%$ of patients present with localized pain in the infected area, and fever was seen in $50 \%$ [3,6,7].

In this case, the initial severe localized tenderness and elevated CRP and ESR were clinically suspect. Nonetheless, infection could not be identified by initial MRI, which led to delayed diagnosis. One way to expedite diagnosis is to perform bacterial culture in conjunction with vertebral bone biopsy. It is thought that pyogenic arthritis of the posterior lumbar facet, as developed in our patient, does not invade the spine. As such, the possibility of negative findings in biopsy is high.
In regard to the dissemination of abscess from vertebral facet joints to adjacent muscles, Magee et al. [1] asserted that the cause was pyogenic arthritis in the facet joint secondary to direct dissemination of abscess within the muscles in the vicinity of the vertebrae, but such dissemination was insufficient to form an abscess in the intracanal epidura.

Baltz et al. [8] and Okada et al. [9] have reported similar experiences. They debrided abscesses disseminated to the intermuscular lesion of the psoas muscle of the anterior aspect of vertebral body, by performing anterior and posterior decompression. In our case, the abscess in the facet joint was located in the dorsal aspect of the epidural space; dissemination of intramuscular abscess was evident only within the muscles, and adequate decompression could only be achieved via the posterior route.

Regarding dissemination of abscess in the facet joint of the vertebrae to the epidural space, Lee et al. [10] reported that good outcome could be achieved using antibiotics cement beads. Our patient was treated by surgical debridement of the abscess followed by intravenous and oral antibiotics, and good outcome was obtained during the follow-up period.

Most pyogenic arthritis of the facet joint is considered to be secondary to spinal osteomyelitis of the vertebral body. Nonetheless, in some cases bacterial invasion of the facet joint by hematogenous dissemination without associated 
vertebral body osteomyelitis have been reported [10]. Based on these experiences, direct bacterial invasion is thought to co-exist with secondary dissemination of osteomyelitis in the vertebral body. Therefore, the diagnosis of pyogenic arthritis in the spinal facet joint is somewhat vague. Even if the initial MRI is unremarkable, imaging should be repeated if symptoms persist or deteriorate. In patients suspected of pyogenic arthritis of the facet joint following physical examination and blood tests, in addition to performing MRI, it is recommended to perform bacterial culture in conjunction with vertebral bone biopsy, supplemented by aggressive antibiotic therapy.

\section{REFERENCES}

1. Magee M, Kannangara S, Dennien B, Lonergan R, Emmett L, Van der Wall H. Paraspinal abscess complicating facet joint injection. Clin Nucl Med 2000;25:71-3.

2. Lee JC, Doh HW, Cho YI, Kim KJ, Kim YI, Shin BJ. Pyogenic arthritis and paraspinal abscess following facet joint steroid injection: a case report. J Korean Soc Spine Surg 2003;10:196-201.

3. Baker AS, Ojemann RG, Swartz MN, Richardson EP Jr. Spinal epidural abscess. N Engl J Med 1975;293:463-8.
4. Darouiche RO. Spinal epidural abscess. N Engl J Med 2006;355:2012-20.

5. Muffoletto AJ, Ketonen LM, Mader JT, Crow WN, Hadjipavlou AG. Hematogenous pyogenic facet joint infection. Spine (Phila Pa 1976) 2001;26:1570-6.

6. Sapico FL, Montgomerie JZ. Pyogenic vertebral osteomyelitis: report of nine cases and review of the literature. Rev Infect Dis 1979;1:754-76.

7. Roberts WA. Pyogenic vertebral osteomyelitis of a lumbar facet joint with associated epidural abscess: a case report with review of the literature. Spine (Phila $\mathrm{Pa}$ 1976) 1988;13:948-52.

8. Baltz MS, Tate DE, Glaser JA. Lumbar facet joint infection associated with epidural and paraspinal abscess. Clin Orthop Relat Res 1997;(339):109-12.

9. Okada F, Takayama H, Doita M, Harada T, Yoshiya S, Kurosaka M. Lumbar facet joint infection associated with epidural and paraspinal abscess: a case report with review of the literature. J Spinal Disord Tech 2005;18:458-61.

10. Lee BJ, Lee SR, Kim ST, Kim TH, Lee SH. Spinal epidural abscess with pyogenic arthritis of facet joint treated with antibiotic-bone cement beads: a case report. Asian Spine J 2007;1:61-4. 